\title{
Progress of China Agricultural Information Technology Research and Applications Based on Registered Agricultural Software Packages
}

\author{
Kaimeng Sun \\ Laboratory of Digital Agricultural Early-warning Technology of Ministry of Agriculture of \\ China, Institute of Agricultural Information of Chinese Academy of Agricultural Sciences, \\ 100081 Beijing, P. R. China
}

\begin{abstract}
By using classified agricultural software products registered during last 15 years from 1994 to 2009, the author analyzed data and statistics in order to look at growth in number of the agro-software packages, their application areas, and research and development organizations, as well as their contribution to the agricultural IT industry in China. Based on the analysis, the article also assessed current agricultural IT sector development and provided some suggestions on further progress.
\end{abstract}

Keywords: Agricultural Information Technology, Applications, Agricultural Modernization.

\section{Introduction}

It is well accepted that development software for agricultural application marks the progress in IT applications in rural development, as well as an important indicator of a country's modern agriculture. Along with fast development of computer technologies in hardware and software, information technology products such as computers, TV, and cell phones have become popular in rural areas in China at a dramatic speed. China's central government, local administrations, and agricultural departments have invested much more in IT in the agricultural sectors. Over the recent past years, computers are more popular and network infrastructure has been development at unprecedented speed in China's rural areas. These provide conditions for IT applications in the agricultural sector, as well as require more on the $\mathrm{R} \& \mathrm{D}$ on agricultural software products. In other words, due to more computers and Internet are used in some rural counties, agricultural software run on the computers becomes an urgent need for IT developers.

China's agricultural IT (AIT) has been developed since 1980s. Early focuses were on agricultural data processing, databases, agricultural expert systems, and crop modeling systems, etc. These applications were generally based on collecting lab data, few directly applied in practical agricultural production and rural lifestyle change. Since the approaching in to IT era, information services for agricultural production, agro product processing, product mobilization, and agricultural supply chains become critical and need software products in providing the services. 
Compared with agricultural IT R\&D team in 20 years ago, it also changed fast. Most of agricultural research institutes and agricultural universities in China have their own developer capacities. Computer technologies are widely applied in their agricultural science research activities becoming popular means. In the mean time, more and more scientific academies, universities, and IT companies are involved in agricultural IT studies and development. Agricultural IT is becoming an area with great potential. As China's vast territory, rich agricultural resources, big agricultural population, and huge contribution of agriculture in the economy, IT applications in agricultural sector will be developing faster in the future.

In order to look at the current status of agricultural software development in China, the author collected data on officially registered 867 agricultural software packages through searching website of the Copyright Protection Center of China (CPCC) by agricultural related key words. The statistics data are further analyzed to reflect current status of AIT applications and development and further indicate the AIT future.

The registered 867 software packages were developed during the past 15 years from 1994 to 2009. During the fifteen years, the number of annual software developed their application areas changed significantly. Partly because of general factors such as increased awareness of copyright protection for software products and computer technology development, the 15 years witnessed progress in agricultural IT application development. From the early in house development to providing AIT services, to today's practical applications in agricultural production and life quality improvement, AIT enters a new era of practical applications.

\section{Quantity of Agricultural Software Packages}

Since the first such software for agricultural applications registered in 1994, the quantity of AIT systems registered grew each year. Especially by 2003, the quantity grew

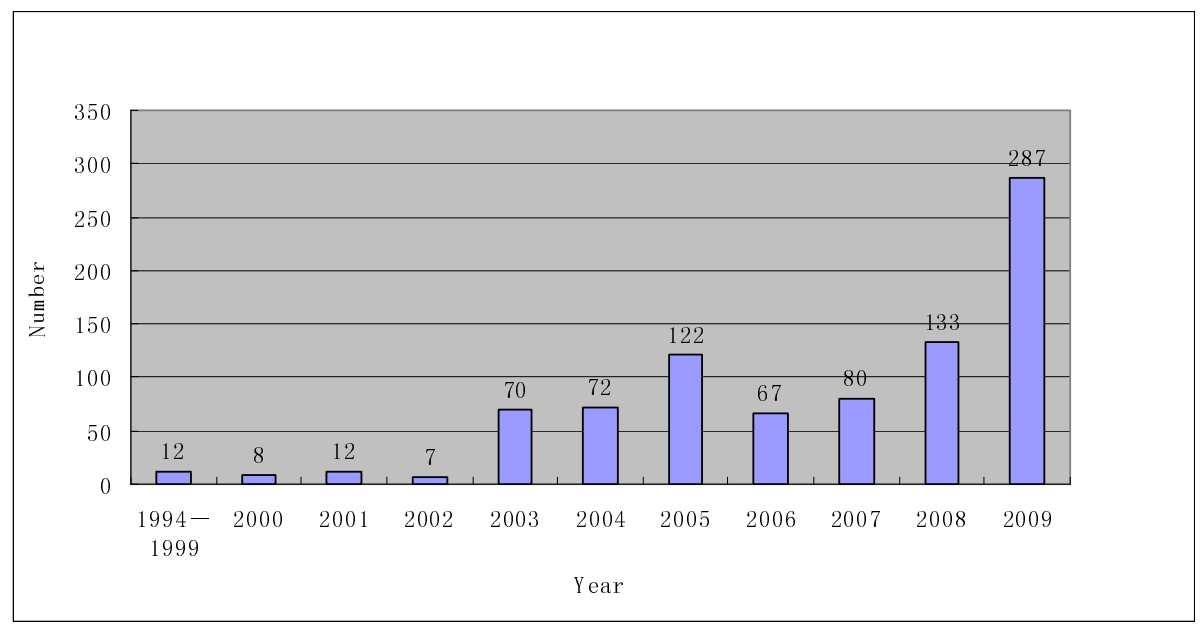

Fig. 1. AIT systems registered over years 
several times. The following figure 1 gives a picture of growth in quantity of the 867 AIT systems registered at China Copyright Protection Center.

Some conclusions can be drawn from the quantity growth:

\subsection{Number of Developed AIT Systems Grow over the Years}

Since 1990s, agricultural information technology has developed fast in China. Particularly since the beginning of 21 st century, as agriculture development obtained strong national policy support, information technology in agricultural sectors has been greatly invested by central and local government which incentivized research and development. Therefore, it is a natural that the number of agricultural software of the agricultural research results increased greatly. Applications of the agricultural information technology has entered to a new development era in terms of increased software packages, improved software quality, and extended application areas.

\subsection{Improved Capacities in Agricultural Software Research and Development}

The increased number of software packages is partly due to more and more developers and research capacities. As a large agricultural sector, Chinese central government paid great attention to the sector investment so that research and development capacities have significantly improved and therefore promoted more agricultural software packages.

\subsection{Improved Awareness of Software Copyright Protection}

With more and more agricultural software products in the market, the awareness of intellectual copyright is improved among researchers and software developers. It is realized gradually that software registration would be one of the best approach to protect developers' efforts and their copyright in the software market. More registered software packages also mark the capacity of developing organizations and software scientists and become an important indicator of scientific research results. Acceptance of intellectual copyrights by the public also encouraged agricultural science research and software development in return.

\section{AIT Applications}

According to the statistics of the registered agricultural software packages, author identified 9 classes of the packages in terms of their application areas: information service for macro agricultural related decision making, agricultural economy management, village management, crop production management, animal husbandry production management, rural business management, agricultural ecological monitoring and control, agricultural information service, and agricultural software tools, mainly including software modules and developing tools. 


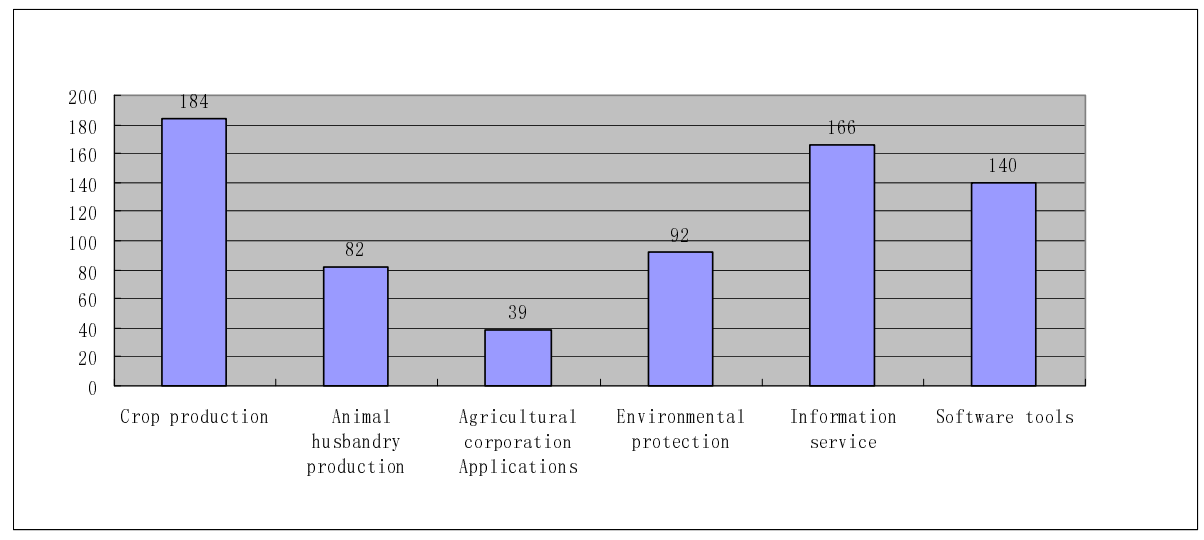

Fig. 2. The distribution of the 867 agricultural software packages in application areas

From the above figure 2, we can draw the following findings:

\subsection{Crop Production Management Software Is Most Widely Applied}

Among the registered agricultural software packages, 187 packages are agricultural crop production management software, or $21.2 \%$, concerning wheat, corn, cotton, rice, beans, and vegetable crops. Functions of the software packages include numerical simulation of crop growing process, planting management, pest diagnose, crop management decision support, and expert systems. As grain and economic crops are main agricultural products in China, computerized information systems are mostly utilized to help solve problems during crop production and become focal point of agricultural research. Since crop production is critical in China's agricultural sector, production decision support software packages take the major market of software products, including decision support on breed selection, fertilizing, irrigation method, optimal yields, pest prevention, field management measures, etc. Crop models, expert systems, and DDS have been developed to practical applications to provide services for agricultural production services. This is very positive trend. Many software packages focus on both agricultural science laws and practical problem solving objectives.

\subsection{More Attention to Agricultural Information Service Functions}

Information service software packages can include agro-product supply, information service, market information, network, and technical training information, etc. Among the survey, this type of software packages take 19.1\%, totaled 166. Because vast Chinese territory are rural areas, many remote countryside communities has poor information access and with very traditional production ways. Through agricultural information service such as Internet and cell phone information dissemination, it helps local farmers to communicate with market and provide useful technical information and exchanges. Their crop product information will also be known through the network by demand side. These systems provide stored data, service functions, and rich market information. It can promote market oriented agricultural production, encourage product 
chain, and help increase farms income so that the agricultural sector will be more effective, convenient, and developed.

\subsection{Environmental Protection Gained More Focus in the AIT Research}

This class of software packages has functions on data processing, analysis, and information service of water resources, soil, and climate data. Natural resources are basis for agricultural production and rural life because all aspect in agricultural production and rural life will depend on local natural conditions. Therefore, the agricultural environment software type focus on functions of climate disaster forecast, field condition monitoring, soil water content analysis, irrigation planning, land uses, etc. The increasing availability of this type of software packages indicate that agricultural conditions get more attention and research on the agricultural environment is going deeper. To make sure favorable agricultural environment will be necessary for prosperous agricultural production and for local farmer income.

\subsection{Software Applied for Animal Husbandry Production Maintains Continuing Progress}

Early in 1980s, computer programs were used for feed formula in China. Software applied for animal husbandry production therefore became one of the earliest applications. Along with the improved computer technologies, animal husbandry software packages develop accordingly. Applications also expanded from the early feed formula to animal husbandry production quality, poultry disease diagnoses, computerized production management, and so on. Especially because of recent fast development, the animal husbandry software packages are targeting large farms and household farmers. Since animal husbandry's high profit than crop farming, they buy these packages. According the survey, there are 82 registered software packages for animal husbandry applications, accounting for $9.5 \%$ of the total in the part 15 years.

\subsection{New Applications for Rural Economy and Village Management}

As China central government focus on agriculture and rural development, daily management in rural areas becomes one of the fast growth in AIT applications. Office automation in rural areas will include financial management, administrative affairs, and material management, especially in financial planning, debt management, clinic operation, farmer management, etc. Application of these software packages make the management in rural areas improved greatly. It helps efficient management. There are a total of 113 rural economy and management software packages surveyed. In particular, rural management software developed very fast since 2005 .

\section{AIT Research and Development Capacities}

\subsection{Agricultural Research Institutes and Universities Are Major Developers of AIT Packages}

According to the statistics over copyright holders of the 867 software packages, 337 or $38.9 \%$ are developed by agricultural academies, 89 packages are developed and owned 
by agricultural universities, accounting for $10.3 \%$. Sum of the two types are about $50 \%$ of the surveyed agricultural software packages. This demonstrates that agricultural research institutes and universities are major developers and take the lead in software research and development.

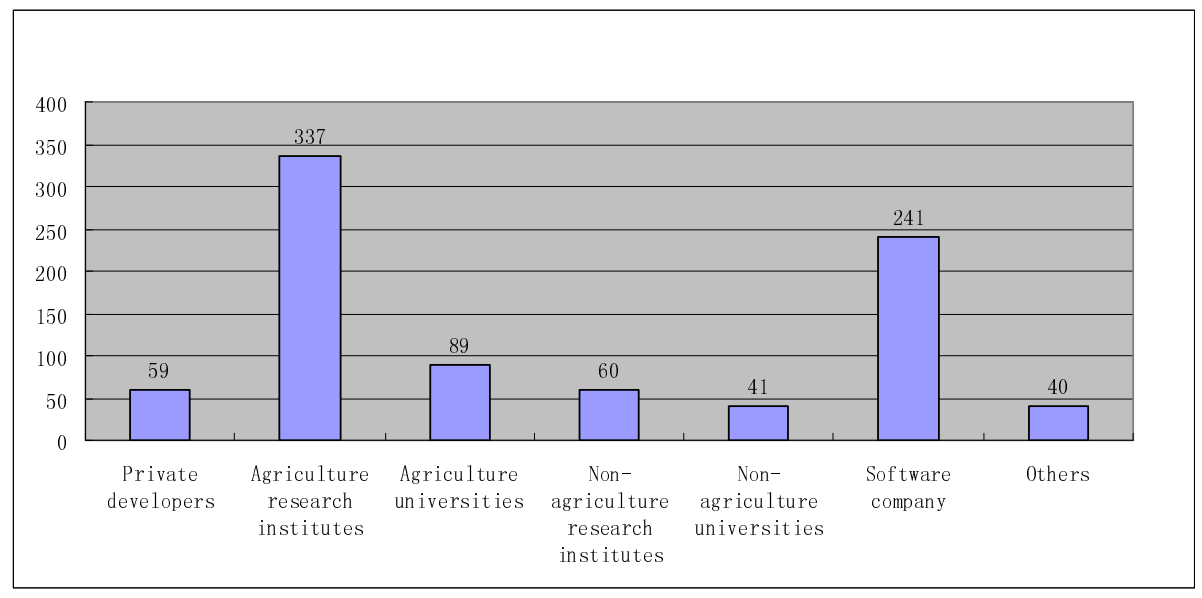

Fig. 3. In the following presents the distribution of the 867 registered agricultural software packages over copyright holders, universities, institutes, and private developers

Among the agricultural research organizations, national and provincial agricultural academies take the lead in terms of rich agricultural data sets, sufficient developer team, excellent computer infrastructures, and large number of various agricultural experts. Meanwhile, local and national financial support also makes them the mainstream of agricultural information technology research and development.

\subsection{The Important Role of Computer Software Companies}

There are 241 software packages were developed by computer software companies, accounting $27.8 \%$ of the total. The author finds out that software companies play important role when end users are not professional in developing the software. Some software companies obtain their developing contract from bidding national and local government projects.

Over the recent years, professional agricultural software development companies grow fast, partly owned by agricultural research organizations and market oriented independent companies specialized in software development. Involvement of the software companies help introduce advanced technologies into the agricultural software market and help improved abundance of the products. At the same time, they also promoted progress of AIT software and benefit both company business and AIT advancement. 


\subsection{More Institutes and Universities Take Part in Agricultural Software Development}

More and more non-agricultural research institutes, colleges, and universities are involved in the AIT R\&D activities, organizations such as Chinese Academy of Sciences, Wuhan University, etc. Some of them develop the software packages through winning bid for national and local projects; some take part in the joint research program. The universities and research institute take their advantages of technical strength and make their contribution in the agricultural studies. This joint development helped focus on agricultural issues, and contributed to competition with agricultural sector capabilities. Of the surveyed software packages, 101 registered software systems were developed and owned by academies and universities, accounting for $11.6 \%$ of the total.

\subsection{Most of the Property Rights Owned by Organizations}

Of the agricultural software package registrations, 808 packages are owned by organizations (93.2\%). In China, these organizations are major developers, including research institutes, universities, software companies, or government departments. Even some privately registered packages may have been funded by some organization. This percentage tells us that most of the AIT products are funded publicly and their objectives are public service oriented. We can affirm that under the current rural economy, it is not practical for AIT project profitable enough. Social benefit is still a major objective.

\section{Conclusions and Recommendations}

From the above research and development status and analysis on the registered agricultural software packages, we can conclude the following in terms of progress and challenges:

\subsection{AIT Develops Successfully in China}

The registered 867 agricultural software packages in the past 15 years demonstrate a fast development. Significant progress has been made in copyright protection, capacity building, and expanded application areas. Due to the increasingly high attention to agricultural sector in China, information technology roles in supporting agricultural production and technical service have become common understanding by researchers and developers. This has changed significantly compared with the situation in 1980s when only few devoting organizations and lack of technical capacities.

\subsection{R\&D on AIT Products Is Developing towards Effectiveness and Efficiency}

In the past, software development focused more on technical advancement but practical applications. Many projects ended with submitted research results, with very weak application support. Now because computers are popular in agricultural sectors, in-

sufficient software systems become a challenge. Current study projects attach great 
importance to demonstration and applications. Therefore, many software packages are developed for such demonstration and application purposes, such that features and functions of the software systems are more local condition oriented, and achieved satisfactory results.

\subsection{R\&D of Agricultural Software Meets New Demands of Today's Agriculture Development}

Like other sectors, agricultural software must follow updated demand on functions. Along with improved agriculture management and farmer education, some critical problems must be addressed in AIT system development.

For instance, early focus on crop models and expert systems in dealing with planting and management must be further improved to solving problems of agricultural product quality. Some recently developed packages focus more on quality and safety. More and more software are using to trace quality in production chain. Again, environmental protection has drown more attentions such that increasingly more software packages are used in analyzing water and land resources, human resource optimization and monitoring.

\subsection{Popularization of AIT Systems Must Be Strengthened}

There are anyway only hundreds of registered agricultural software packages. This is far insufficient in terms of China's large rural area and agricultural population. Even of the current software packages, very limited number of packages is suitable for agricultural production and rural population demands. Many such packages need to be improved for better agricultural applications. Therefore, central and local governments need to apply and popularize the existing products. If the heavily invested current systems were not fully applied, it will be a huge waste. Though some specially planned demonstration and application projects, it is not enough. Further investment shall include both capital and human resources to improve the effectiveness and efficiency of agricultural software applications. Otherwise AIT progress will be not as expected.

Promotion of agricultural software needs joint efforts by government bodies, research institutes and rural users by working together closely. Under the current situation, sales of the AIT software systems can hardly profitable. Therefore, capital support from government will be needed for software development, technical training, and hardware investment. Only by these supports, agricultural software can be applied effectively and efficiently.

Currently, improving applications of existing agricultural software packages is an important task for promoting AIT applications. Only by increasingly more applications, can the research and development of AIT systems progress at a sound basis. In return, the national and local investment will be paid off and AIT development progressed. This will facilitate AIT to provide greater contribution to agriculture production and to farmers' technical service. And finally it will contribute to modern agriculture in China. 


\section{Acknowledgement}

This research was supported by National Scientific and Technical Supporting Programs Funded by Ministry of Science and Technology of China(2006BAD10A06, 2006BAD10A12), Special Fund of Basic Scientific Research and Operation Foundation for Commonweal Scientific Research Institutes (2008J-1-06).

\section{Reference}

Copyright Protection Center of China (CPCC),

http: / /www. ccopyright.com.cn/cpcc/ 